\title{
A MICROFLUIDIC PLATFORM FOR THE FLUIDIC ISOLATION AND OBSERVATION OF CELLS CHALLENGED WITH PATHOGENS
}

\author{
C. D. James ${ }^{1}$, M.W. Moorman ${ }^{1}$, B.D. Carson ${ }^{1}$, J. Joo' , C.S. Branda ${ }^{3}$,

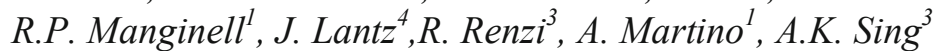 \\ ${ }^{1}$ Sandia National Laboratories, Albuquerque, NM, USA \\ ${ }^{2}$ University of Tennessee, Knoxville, TN, USA \\ ${ }^{3}$ Sandia National Laboratories, Livermore, CA, USA \\ ${ }^{4}$ GAITS, Albuquerque, NM, USA
}

\begin{abstract}
Single-cell analysis offers a promising method of studying cellular functions including investigation of mechanisms of hostpathogen interaction. We are developing a microfluidic platform that integrates single-cell capture along with an optimized interface for high-resolution fluorescence microscopy. The goal is to monitor, using fluorescent reporter constructs and labeled antibodies, the early events in signal transduction in innate immunity pathways of macrophages and other immune cells. The work presented discusses the development of the single-cell capture device, the iCellator chip, that isolates, captures, and exposes cells to pathogenic insults. We have successfully monitored the translocation of $\mathrm{NF}-\mathrm{kB}$, a transcription factor, from the cytoplasm to the nucleus after lipopolysaccharide (LPS) stimulation of RAW264.7 macrophages.
\end{abstract}

\section{INTRODUCTION}

Our team at Sandia National Laboratories has developed a microfluidic system to facilitate single cell imaging and analysis. This system is currently being utilized for real-time monitoring of fluorescent reporter constructs and labeled antibodies to examine the early events of signal transduction in the innate immune response. Traditional methods to study this pathway provide population averaged measurements, and thus it is difficult to interpret individual cell responses because not all cells in a population reside in the same initial physiological state or undergo the same degree of infection. Further, cell-intrinsic and extrinsic events cannot be separated when groups of cells are exposed in bulk culture (cytokines, cell-cell interaction). By isolating individual cells, we are able to perform real-time, quantitative analysis of cell signaling cascades involved in host-pathogen interactions, with a special emphasis on the early events that occur during immune response.

The system consists of two components: a microfluidic platform, called the iCellator chip, that enables single cell capture, isolation, challenge, and high-resolution microscopy, and an instrument for fluid handling and platform temperature control that uses a simple computer interface. Figure 1 shows a schematic of the iCellator chip design. Other work has demonstrated single cell capturing techniques optimized for optical interrogation such as DiCarlo [1] and Tourovskaia [2], but neither approach has achieved complete cell isolation as this effort has.

\section{iCELLATOR DEVICES}

The chip design described here is an improvement over earlier prototype designs that we have reported in [3]. Compared to these earlier designs, the more recent designs feature more efficient cell trapping structures, better cell isolation, an improved imaging interface, and dual imaging chambers. The dual imaging chambers allow us to run simultaneous control and challenge experiments on the same chip. Each chamber contains 150 fluidically isolated traps where the cells are captured by micron-scale constrictions.

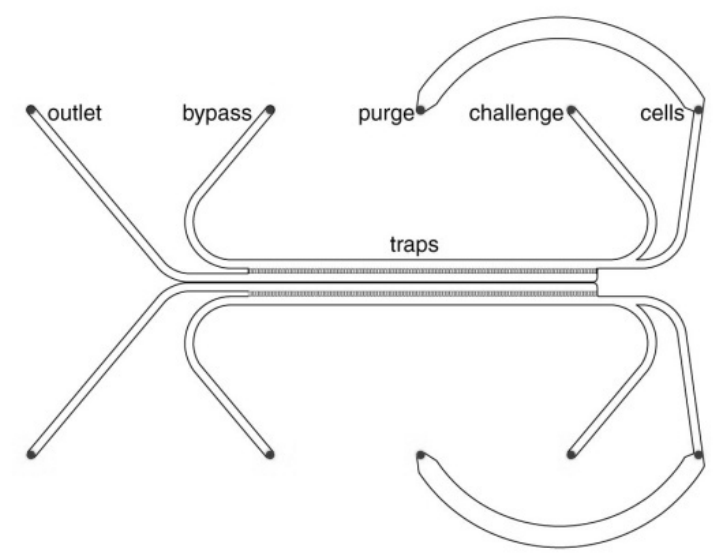

Figure 1: Schematic of the iCellator chip showing challenge and cell loading inlets, trap location, and outlets with separated imaging chambers to allow challenge and control experiments.

These cell constrictions are semi-circular to allow the cell to easily conform to its shape. The constrictions place cells approximately 3 um immediately beneath the coverslip lid for low background fluorescence imaging, unlike the prior design which had a tendency to trap cells in the middle of the flow channels, approximately 15 um below the surface of the coverslip. Figure 2 shows a schematic and optical image of our most recent trapping structures.
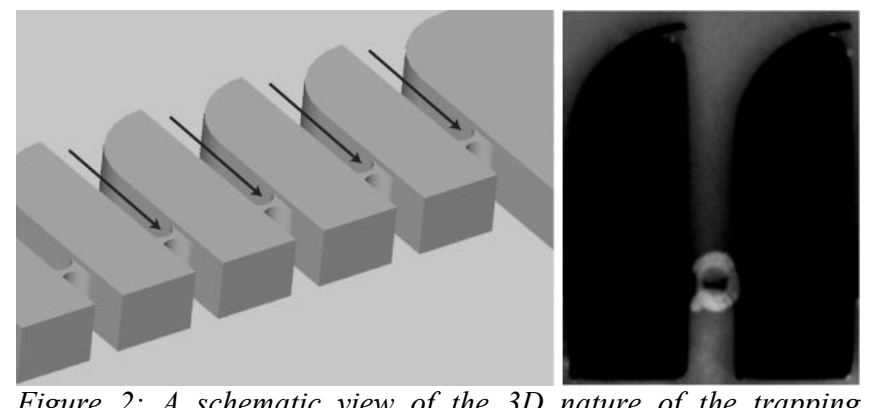

Figure 2: A schematic view of the $3 D$ nature of the trapping features, with arrows showing the direction of fluid flow into the traps. The adjacent fluorescence image shows a RAW264.7 cell on a trapping feature.

Computational modeling was used to inform the design of the new cell trap structures. Placement of the constrictions at the end of long channels was done to better control the fluidic isolation of each cell trap. Once trapped, the combination of continuous fluid flow and architecture isolates each trapped cell from the secreted cytokines of other cells. This enables study of the effects of paracrine signaling on long-term host cell response to pathogenic 
challenges. Figure 3 shows the results of fluidic modeling on the previous cell trap structures. These results show that the prior design had a critical trap depth, measured by the value $\mathrm{Di}$, which indicated how deep into the trap a cell must lie in order to be isolated. Above the Di height the cell could be exposed to cytokine secretions from other cells. The Di height for the majority of the traps was less than half of the overall trap height $\mathrm{D}$, indicating a large area of each trap was not fluidically isolated.

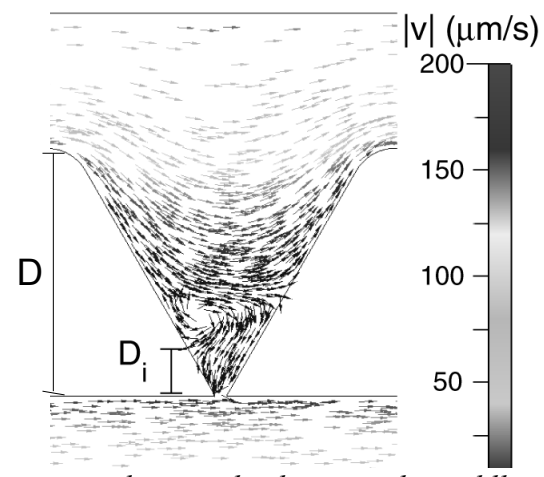

Figure 3: Flow streamlines in the device at the middle trap. The trap depth (D) and the depth of isolation (Di) are noted.

Similar fluidic modeling was performed on the new trapping structures. This modeling showed that the Di value was essentially equal to the length of the channels leading to the cell constrictions. Figure 4 shows results of this modeling. This meant that not only were captured cells much more likely to be fluidically isolated, but also that there was a greater overall uniformity of Di values across all the cell trap locations, which made for better experimental conditions overall.
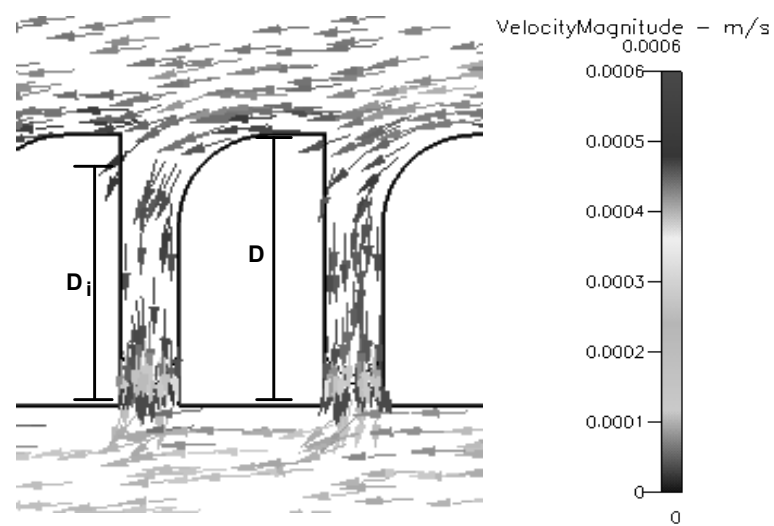

Figure 4: Flow streamlines in the middle trap devices showing a nearly equivalent trap depth (D) and depth of isolation (Di).

\section{iCellator Fabrication}

The devices followed a fairly simple microfabrication process. All chips were fabricated using 6-in. $450 \mu \mathrm{m}$ thick double polished silicon wafers. A series of deep reactive ion etch (DRIE) steps on the front-side of the wafer defined the device channels and trap structures, while a through-wafer backside DRIE defined the $200 \mu \mathrm{m}$ diameter inlet and outlet ports. After an $\mathrm{O} 2$ plasma $(150 \mathrm{~W}$ for $30 \mathrm{~s}$ ) and acetone sonication to remove resist, wafers were then diced into individual die. No. 1.5 Pyrex coverslips were then bonded to the silicon chips using a custom-made anodic bonder system. Excess glass was trimmed from the die before being placed into the chip's testing manifold.

\section{TESTING SETUP}

Figure 5 shows an iCellator chip installed in a manifold that provides heating, fluid exchange, and a low-profile optical interface enabling high-resolution oil-immersion imaging with upright or inverted microscopes. The instrumentation for fluid

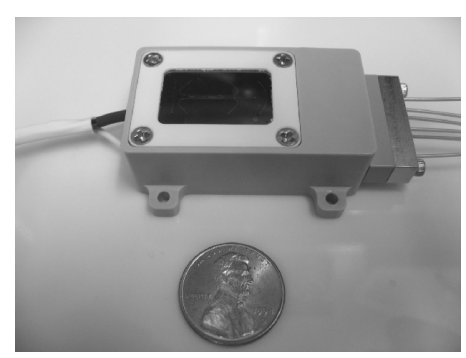

Figure 5: PEEK fixturing featuring fluidic interconnects, integrated heating, and a compression frame to seal the chip against the manifold.

handling and temperature control consists of a pneumatic and electrical control module joined by an umbilical cord to heated fluid reservoirs and valving. The larger control module can be placed near the workspace, while necessary fluid reservoirs can be mounted directly on the microscope stage to minimize swept fluid volumes. Fluid control is provided by six pneumatic modules, two high-flow and four low-flow, that provide a flow rate through the chip of $0.5-110 \mu \mathrm{l} / \mathrm{min}$. The temperature control system can maintain a range of cell environmental temperatures, from room temperature to boiling, to within $\pm 0.3{ }^{\circ} \mathrm{C}$. The stage-mounted fluid reservoirs are built to accept commercially available microcentrifuge tubes for ease of solution preparation and transport, while the valving is based on a novel design that minimizes fluid pressure pulses during valve actuation. Using these innovative features, the system pictured in Figure 6 provides precise control of the on-chip cellular environment.
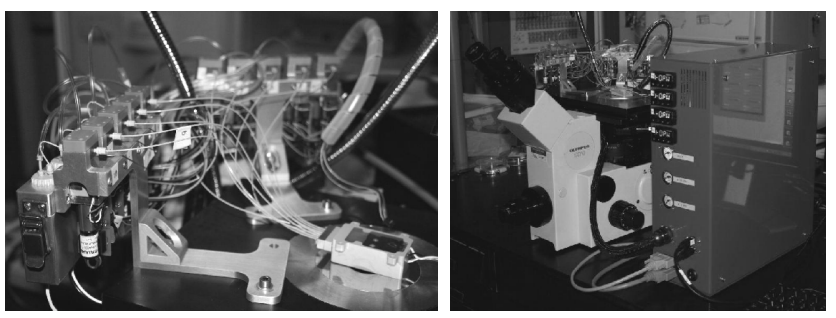

Figure 6: Fluid control reservoirs and valves mounted on microscope stage. The adjacent image shows the flow control box with pressure umbilical connection.

\section{EXPERIMENTATION}

Experiments with the chip have thus far utilized the RAW 264.7 mouse macrophage line. Testing begins by flushing the chip and manifold with endotoxin-free water then medium. The total volume of a single imaging chamber is $\sim 60 \mathrm{~nL}$, thus enabling rapid 
medium exchange within the device. After this cleaning/passivation step, the cells are flowed into the device and captured at the cell constrictions. Prior to their use on the iCellator chip the cells, which have a fluorescent reporter construct (GFPRelA), are stained with Hoechst 33258 (nuclear) and propidium iodide (live/dead). Figure 7 demonstrates a typical cell capture.

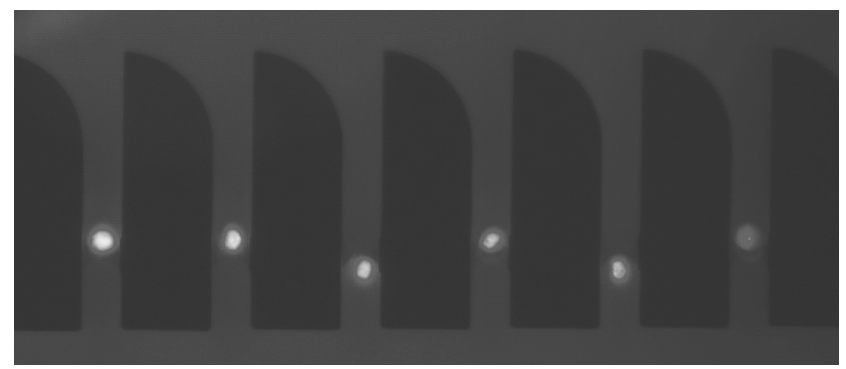

Figure 7: Hoechst stained single cells after trapping in the iCellator chip. Fluid flow is coming from the top of the image.

Once captured, the cells can be maintained in the chip for extended periods of time, requiring only temperature maintenance of the fluid manifold and steady perfusion of medium. The longest incubation period of the cells thus far has been approximately a 3 day long experiment. During longer duration experiments it is not uncommon to record cell division, as shown in Figure 8.
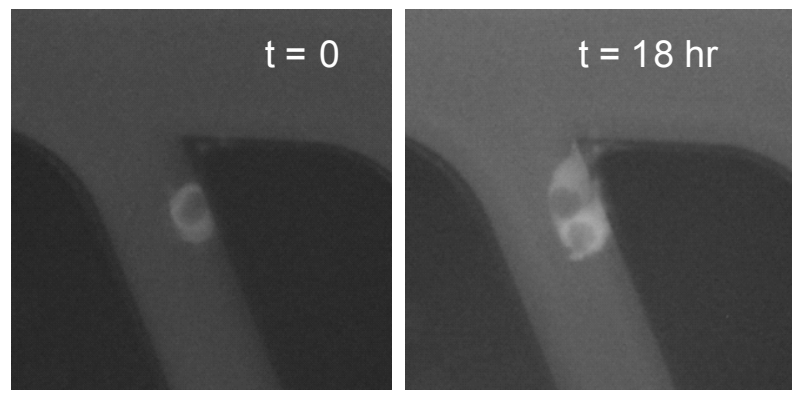

Figure 8: Division of cell adhering to entrance channel of a trap during an extended duration test.

After capture, a short incubation of $\sim 0.5 \mathrm{hr}$ is performed to assure the cells are healthy and uncontaminated. This is verified by monitoring the propidium iodide live/dead stain and looking for premature translocation events. Once this is verified, the challenge channel can be exposed while the control channel is left unexposed. Challenges we have used include live $E$. coli bacteria and LPS purified from $E$. coli and other bacteria.

\section{RESULTS}

After challenge, we monitor the translocation of RelA, a subunit of the transcription factor $\mathrm{NF}-\mathrm{kB}$, from the cytoplasm to the nucleus. Figure 9 shows a trapped macrophage with GFP labeled RelA. Figures 9a-c show GFP:RelA translocation progress after cell exposure to purified $E$. coli LPS. In figure 9d are image analysis results showing GFP fluorescence as a function of position and time. The dashed line on Figure 9a shows the location where the intensity measurements were taken. On-chip studies of these responses have lasted for $\sim 3$ days with greater than $95 \%$ cell viability at conclusion.
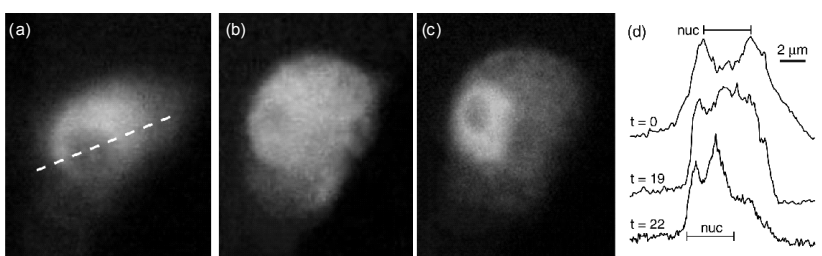

Figure 9: GFP:RelA translocation in a trapped $R A W$ cell at $t$ $=-12(a), 7(b)$, and 10 min (c). The cell was challenged with 1 $\mu M$ E. coli LPS at $t=0$. (d) The intensity of GFP:RelA fluorescence in the cell as a function of position and time.

In our prior work, we demonstrated that a high concentration LPS challenge led to an early and rapidly completed GFP:RelA translocation in host cells, while a lower concentration challenge led to a delayed and slowly completed translocation. While simple translocation events, such as the one described in the figure above were recorded, our work also revealed the possibility of more complex kinetic events involving the oscillation of $\mathrm{NF \kappa B}$ within the cell. Figure 10 shows the analysis of a cell that has undergone oscillation behavior in response to an $E$. coli LPS challenge.

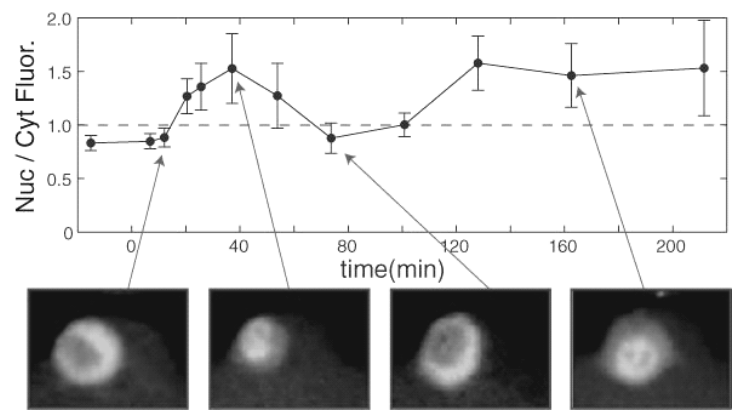

Figure 10: Oscillation of RelA in an iCellator-captured single cell challenged with E. coli LPS as a function of time. The $\mathrm{Nuc/Cyt}$ axis represents the ratio of GFP:RelA imaged in the nucleus to the cytoplasm.

Analysis of the GFP:RelA in the nucleus and the cytoplasm shows that not only is the oscillation phenomenon widespread, but that individual cells translocate and oscillate in unique manners. The oscillation magnitude and rate of response has also been found to depend not only on LPS dose but its chemotype (i.e. source species). These laboratory observations of oscillation agree with our computational modeling efforts of simulated immune response which predicted NF- $\mathrm{BB}$ oscillations after LPS exposure. Figure 11 shows the four general types of oscillation behavior predicted. Specifics on the computational modeling effort will be detailed in another forum.

Figure 12 shows a chart comparing the oscillation behavior of nine single cells to the same $E$. coli LPS exposure. Analysis of the oscillation patterns reveals heterogeneity in the oscillation behavior of each of the cells. This is demonstrated by asynchrony in timing of the first translocation event compared to subsequent translocations. In this specific experiment, it was observed that the percentage of single cells undergoing an initial translocation event in response to LPS is high ( $\sim 90 \%)$, however the percentage of cells that undergo a secondary translocation event is somewhat lower 

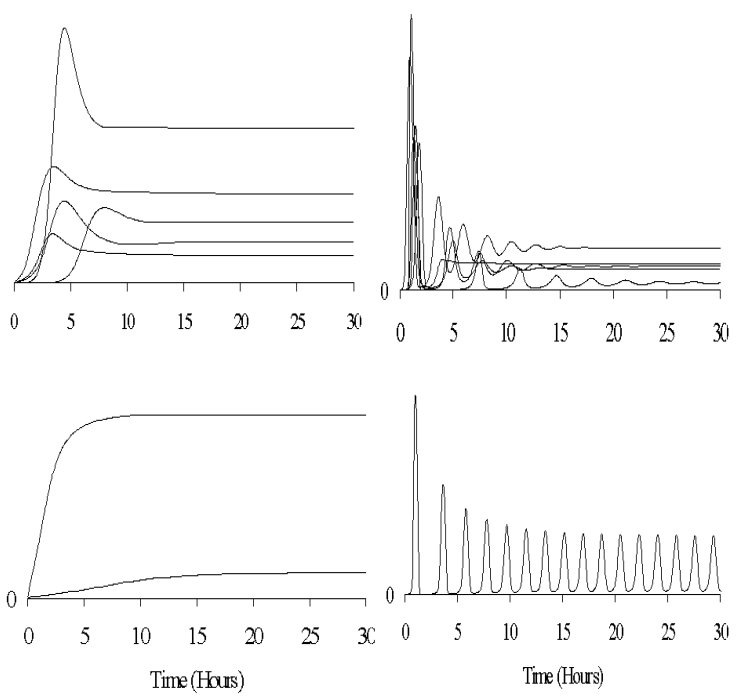

Figure 11: Four general classes of $N F \kappa B$ oscillation behavior as predicted by computational modeling.

( $53 \%)$. Additionally, cells are shown to exhibit the different oscillation patterns predicted by our computational modeling efforts. The oscillation patterns shown in Figure 12 reveal the manifestation of the top two generalized patterns predicated by our modeling, though evidence of all four of the patterns have been observed in other experiments.

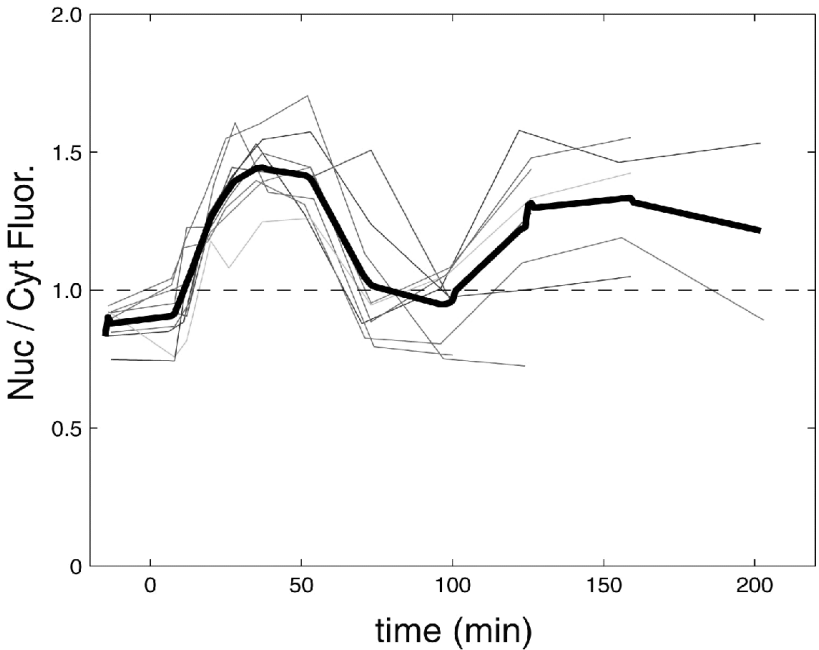

Figure 12: Compiled oscillation behavior of nine single cells responding to the same E. coli LPS challenge. The thick line indicates a population averaged measurement.

\section{CONCLUSIONS}

We have developed an integrated microfluidic platform for imaging and analysis of host-pathogen interactions with single cell resolution - validated by performing LPS and bacterial challenge experiments on macrophages. Using reporter constructs and labeled antibodies, we observed NF- $\mathrm{KB}$ translocation events and oscillations in localization (nucleus vs cytoplasm) for the first time. TLR-4 signaling kinetics in macrophages were quantified as a function of LPS dosage, and it has been found that high concentrations lead to an early, rapid, and synchronous translocation response, while low concentrations produce a delayed and less synchronous response. Our work with computational models of the immune response predicated oscillation patterns of the NF- $\kappa \mathrm{B}$ in response to LPS challenge, which has been clearly observed at the single cell level. Single cell measurement at this level provides unique insight into the operation of the global signaling network, and will be a valuable tool in studying the role of cell-intrinsic and extrinsic events in innate immunity.

\section{FUNDING STATEMENT}

Sandia National Laboratories is a multi-program laboratory operated by Sandia Corporation, a wholly owned subsidiary of Lockheed Martin company, for the U.S. Department of Energy's National Nuclear Security Administration under contract DEAC04-94AL85000.

\section{COPYRIGHT NOTICE}

This material has been authored by Sandia National Laboratories under Contract No. DE-AC04-94AL85000 with the U.S. Department of Energy. The United States Government retains and the publisher, by accepting the material for publication, acknowledges that the United States Government retains a nonexclusive, paid-up, irrevocable, world-wide license to publish or reproduce the published form of this manuscript, or allow others to do so, for United States Government purposes.

\section{REFERENCES}

[1] D. Di Carlo et al, "Single-cell enzyme concentrations, kinetics, and inhibition analysis using high-density hydrodynamic cell isolation arrays", Analytical Chemistry, Vol. 78, 2006.

[2] A. Tourovskaia, et al, "Differentiation-on-a-chip: A microfluidic platform for long-term cell culture studies", Lab on a Chip, Vol 5, 2005.

[3] James, C.D. et al, "Nuclear translocation kinetics of NF- $\kappa$ B in macrophages challenged with pathogens in a microfluidic platform”, Biomedical Microdevices, 11, 2009.

\section{CONTACT}

Conrad James, cdjame@sandia.gov

Sandia National Laboratories

Biosensors and Nanomaterials PO Box 5800, MS 0892

Albuquerque, NM 87185-0892 